\title{
Numerical Study on Shock Train Characteristics in Divergent Channels
}

\author{
P. S. Vignesh Ram, T. H. Kim and H. D. Kim ${ }^{\dagger}$ \\ Department of Mechanical Engineering, Andong National University, Andong, 760-749, South Korea \\ † Corresponding Author Email: kimhd@anu.ac.kr
}

(Received August 7, 2019; accepted December 8, 2019)

\begin{abstract}
In any supersonic intake, the flow decelerates from supersonic to subsonic speed through a constant or divergent channel "isolator" by a series of bifurcated compression shock waves referred to as a shock train. It is important to understand the characteristics of the shock train which occur inside the isolator to improve the performance of scramjet engines. In the present work, numerical simulations were carried out to investigate the characteristics of the shock train occurring in the divergent channels using coupled implicit Reynolds Averaged Navier-Stokes (RANS) equations along with the two-equation k-w SST turbulence model. Results show that the downstream pressure variation causes the shock train length to decrease and the shock structure phenomenon varies from Mach reflection to Regular reflection. The variation of the inlet Mach number has less influence on the shock train length and the location of the shock train is determined by the area ratio. In comparison with the constant area duct, the shock train structure phenomena varies from Mach reflection to regular reflection in the divergent channel. Also, the increase in divergent angle raises the total pressure loss.
\end{abstract}

Keywords: Shock boundary layer interaction; Pseudo shock wave; Separated flows; Shock reflection phenomenon.

\section{NOMENCLATURE}

$\begin{array}{ll}B & \text { blockage ratio } \\ C_{p} & \text { specific heat at constant pressure } \\ D_{e q} & \text { equivalent diameter } \\ D_{\omega} & \text { cross diffusion term } \\ E & \text { energy } \\ G_{k}, G_{\omega} & \text { turbulence production term } \\ k & \text { turbulent kinetic energy } \\ L & \text { length } \\ L_{p s w} & \text { pseudo shock length } \\ M & \text { Mach number } \\ p & \text { pressure } \\ P_{r t} & \text { Prandtl number } \\ R_{e \theta} & \text { Reynolds number } \\ u_{i,}, u_{j}, u_{k} & \text { velocity components } \\ X, Y, Z & \text { Cartesian coordinates }\end{array}$

\section{INTRODUCTION}

A scramjet (supersonic combustion ramjet) is a variant of a ramjet engine which is vital for any hypersonic flight. The scramjet engine is comprised of an inlet, an isolator, a combustor, and a nozzle. The schematic of typical scramjet engine is shown in Fig.1. The flow compression takes place inside the

$\begin{array}{ll}\rho & \text { density } \\ \delta_{i j} & \text { Kronecker delta } \\ \delta & \text { boundary layer thickness } \\ \delta^{*} & \text { displacement thickness } \\ \Delta_{k,}, \Delta_{\omega} & \text { turbulence dissipation term } \\ \theta & \text { momentum thickness } \\ \mu & \text { dynamic viscosity } \\ \mu_{e f f} & \text { effective viscosity } \\ \mu t & \text { turbulent viscosity } \\ \xi & \text { divergent angle } \\ \omega & \text { specific dissipation rate } \\ \varepsilon & \text { dissipation rate } \\ \tau_{i j} & \text { stress tensor }\end{array}$

inlet and isolator region by means of a series of shock wave structures. The isolator prevents the pressure rise due to combustion from reaching the inlet region (Heiser and Mehta 1994). In the absence of the isolator, a small downstream pressure disturbance can affect the inlet performance and may lead to unstart condition. For the past several decades many experimental and numerical investigations have been 


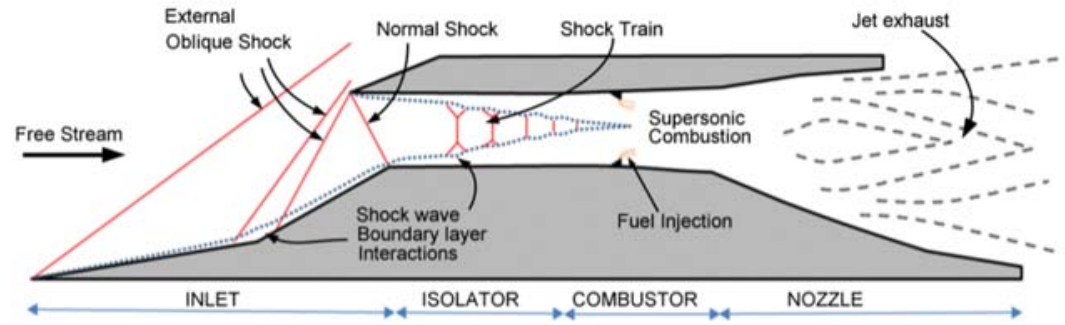

Fig. 1. Schematic of typical scramjet engine.

conducted on the start/unstart phenomenon of hypersonic intake (Wagner et al. 2009; Zhang et al. 2016; Hutchins et al. 2014, Chang et al. 2014, Das and Prasad 2010). Thus to prevent inlet un-start, the isolator plays a critical role in the scramjet engine.

The compression shock inside the isolator can be classified into four different possible configurations shown in a constant area duct illustrated in Fig.2. For freestream Mach number $\mathrm{M}_{1 \mathrm{e}}<1.2$, the shock structure is close to an inviscid normal shock. With the increase in the Mach value to 1.3 , a weak interaction between the shock wave and the boundary layer takes place and the shock wave is curved as shown in Fig. 2 (b). With a further increase in $\mathrm{M}$, the foot of the shock wave separates and producing a lambda shaped structure with a tendency to reattachment as shown in Fig.2(c). For $\mathrm{M}_{1 \mathrm{e}}>1.5$, the shock bifurcates into several shocks and is referred to as a "shock train". The pressure continues to rise downstream of the shock train for a certain distance often called a "mixing region". The entire shock train and mixing region together is referred to as "pseudo-shock wave" (Matsuo and Kim 1999a; Gnani et al. 2016). This pseudo-shock wave system is mainly influenced by the geometrical parameters, the upstream Mach number, the flow confinement and the upstream to downstream pressure ratio (Om et al. 1985). The upstream boundary layer can shift the location and shape of the shock train. This can be related in terms of flow confinement (Matsuo and Kim 1999a) which is defined as the ratio of the undisturbed boundary layer thickness $(\delta)$ to the duct half height(h).

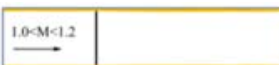

(a) Normal shock wave

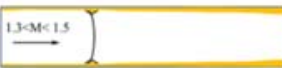

(c) Lambda shock wave Fig. 2. Schematic of formation of pseudo shock wave in a constant area duct (Matsuo and Kim 1999a).

$\frac{L_{p s w}\left(M^{2}-1\right) R e_{\theta}^{(1 / 4)}}{D^{1 / 2} \theta^{1 / 2}}=50\left(\frac{p_{2}}{p_{1}}-1\right)+170\left(\frac{p_{2}}{p_{1}}-1\right)^{2}$

Carroll and Dutton (1990) revealed that the length of the shock train increases with increases in confinement level. Also, the transition of a leading shock from a $\lambda$ shaped to an $\mathrm{x}$-shaped structure is observed when increasing the confinement level. The increase in length of pseudo shock waves by confinement is also noticed by Om and Childs (1985) through an experiment conducted in a circular duct. The researchers also postulated a one-dimensional flow model to explain the formation of a series of normal shock waves. Numerous studies apply theoretical and analytical models to determine the length of the pseudo shock wave (Om and Childs 1985; Waltrup and Billig 1973; Ikui et al. 1974; Ikui et al. 1981; Ikui et al. 1980; Matsuo et al. 1999a). Waltrup and Billig (1973) presented a simple empirical relation (Eq.1) to determine the pseudo shock length. This equation can also be used to describe the wall pressure distribution of the interaction region. Ikui et al.(1974) proposed the diffusion model to predict the length of the pseudo shock wave, which refines the shockless model developed by Tamaki et al.(Tamaki et al. 1970; Tamaki et al. 1971). Likewise, the mass averaging pseudo-shock model for a constant area duct with a fully turbulent boundary layer is proposed by Matsuo et al. (1999b). This model predicts the downstream flow properties of the pseudo-shock solely from the upstream mass averaged values. Among the above models, Waltrup's (Waltrup and Billig 1973) empirical equation used widely in predicting the length of the pseudo shock wave. But the pressure deviates from the experimental measurement observed by Weiss et al. (2010). This occurs because the empirical model does not account for the mixing region. Weiss et al. (2010) confirmed that the position and the length of the pseudo shock wave is dominated by the Mach number and confinement level. He also explained that the change in Reynolds number, $R_{e_{\theta}}$ in Eq.1 is negligible when compared with Mach number which changes by the power of two. Many researchers have extensively studied pseudo shock waves, yet many flow features remain undiscovered like the shape transition of pseudo shock waves, the separation region and the oscillation of pseudo shock waves due to pressure variation. The occurrence of a pseudo-shock wave in a shock tube utilizes the computational fluid dynamics method by Kim et al. (2017). The variation in shock strength and its characteristics are greatly influenced by tube diameter and the pressure ratio. Quaatz et al. (2014) suggested that these complex flow field are difficult to analyze by experiment alone. This led researchers to study using 
computational analysis. The Reynolds Averaged Navier-Stokes (RANS) equations are widely used to solve complex flow features. RANS equations are sensitive towards a turbulence closure model. To predict the complex flow field, an understanding of the turbulence model effectiveness is required. Sun et al. (2003) conducted numerical studies on pseudoshock waves in a rectangular duct at $\mathrm{M}=2.0$ and $R_{e_{\infty}}$ $=2.53 \times 10^{7}$ applying the Bladwin-Lomax turbulence model with corresponding results to those in experiments. The advantage of the Baldwin-Lomax model is low computational cost while the main drawback is the assumption that turbulence is isotropic and with a decreased effect in predicting the separated flows. The standard $k-\varepsilon$ model is a semiempirical model based on transport equations for turbulent kinetic energy $(k)$ and dissipation rate $(\varepsilon)$. Huang et al. (2011) employed the standard k- $\varepsilon$ model to study the flow field inside the scramjet isolator. Here too, their results agree with the experimental results (Sun et al. 2003). Gnani et al. (Gnani et al. 2018b; Gnani et al. 2018a) investigated shock train characteristics and their movements under forced back pressure oscillation using 2-dimensional RANS with a $k-\omega$ model. Upon using various turbulence models he concluded that the $k-\omega$ Wilcox model compares more favorably with the experimental results (Sun et al. 2003). The main problem with the Wilcox model is a strong dependence on the freestream condition. The results can abruptly vary with initial $\omega$ value at the inlet. To overcome this problem a limiter is set to the eddy-viscosity formulation with the resulting advantage of $k-\omega S S T$ models are their ability to accurately predict the onset and the amount of flow separation under adverse pressure gradients Menter (1994). Gounko and Mazhul (2018) simulated the flow with a pseudo shock in an axisymmetric expanding duct with a frontal in-let at $\mathrm{M}=6.0$ using $k-\omega-S S T$ model. He determined the end location of the pseudo shock in a cylindrical divergent duct will be the location at which the turbulent viscosity $\left(\mu_{\mathrm{t}}\right)$ is maximum.

The present study analyses the characteristic of the shock train in the divergent channel numerically using the RANS equation along with the mentor SST model. The effect of back pressure, Mach number and the divergent angle on the shock train is observed. Though the shock train flow is more complex in a three-dimensional environment, two dimensional flow demonstrates how the side wall has less influence on the symmetry plane. Also the present study includes an inquiry of the nature of the shock train considering the influence of the side wall. The study also uses the shock polar analysis to investigate shock reflection characteristics. Finally, the effect of divergent angle on total pressure loss is investigated.

\section{COMPUTATIONAL METHODOLOGY}

\subsection{Governing Equation}

The present study carried out both $2 \mathrm{D}$ and 3D numerical simulations. Favre-averaged Navier-
Stokes equations were used to determine the conservation of mass, momentum, and energy and along with $k-\omega$ turbulence model are solved. The governing equations in the tensor notations are as follows,

$\frac{\partial \rho}{\partial t}+\frac{\partial\left(\rho u_{i}\right)}{\partial x_{i}}=0$

$\frac{D\left(\rho u_{i}\right)}{D t}=-\frac{\partial p}{\partial x_{i}}+\frac{\partial}{\partial x_{j}}\left[\mu_{e f f}\left(\frac{\partial u_{i}}{\partial x_{j}}+\frac{\partial u_{j}}{\partial x_{i}}-\right.\right.$

$\left.\left.\frac{2}{3} \delta_{i j} \frac{\partial u_{k}}{\partial x_{k}}\right)\right]+\frac{\partial\left(-\rho \overline{u_{\imath}^{\prime} u_{j}^{\prime}}\right)}{\partial x_{j}}$

$\frac{\partial(\rho \mathrm{E})}{\partial t}+\frac{\partial\left(\rho u_{j} \mathrm{E}+u_{j} p\right)}{\partial x_{j}}=\frac{\partial}{\partial x_{j}}\left[\left(\alpha+\frac{C_{p} \mu_{t}}{P r_{t}}\right) \frac{\partial T}{\partial x_{j}}+\right.$

$\left.u_{i}\left(\tau_{i j}\right)_{e f f}\right]$

where $E$ and $T$ are the mass averaged values and $\left(\tau_{i j}\right)$ is the shear stress tensor, which is defined as,

$\tau_{i j}=\mu_{e f f}\left(\frac{\partial u_{i}}{\partial x_{j}}+\frac{\partial u_{j}}{\partial x_{i}}-\frac{2}{3} \delta_{i j} \frac{\partial u_{i}}{\partial x_{i}}\right)$

The equation of state for perfect gas is added to close the system. In the present work, the $k-\omega S S T$ Menter (1994) turbulence model is used. The transport equation for turbulence kinetic energy $(k)$ and the specific dissipation rate $(\omega)$ are as follows,

$\frac{\partial(\rho k)}{\partial t}+\frac{\partial\left(\rho k u_{i}\right)}{\partial x_{i}}=\frac{\partial}{\partial x_{j}}\left[\left(\mu+\frac{\mu_{t}}{\sigma_{k}}\right) \frac{\partial k}{\partial x_{j}}\right]+G_{k}-\Delta_{k}$
$\frac{\partial(\rho \omega)}{\partial t}+\frac{\partial\left(\rho \omega u_{i}\right)}{\partial x_{i}}=\frac{\partial}{\partial x_{j}}\left[\left(\mu+\frac{\mu_{t}}{\sigma_{\omega}}\right) \frac{\partial k}{\partial x_{j}}\right]+G_{\omega}-\Delta_{\omega}+$

where $G_{k}$ represents the generation of turbulence kinetic energy due to mean velocity gradients. The $G_{\omega}$ accounts for the production of $\omega . \Delta_{k}$ and $\Delta_{\omega}$ represent the dissipation of $k$ and $\omega$ due to turbulence, respectively. $\sigma_{k}$ and $\sigma_{\omega}$ are the turbulent Prandtl numbers for $k$ and $\omega$, respectively. The numerical simulations were carried out using the commercial CFD code ANSYS Fluent. A 3-D halfdomain block-structured grid was created using ICEM CFD. The RANS equations are discretized using the gauss cell centered finite volume method. The inviscid and viscous fluxes are evaluated using an AUSM flux vector splitting scheme based on second order upwinding scheme.

\section{2 Numerical Setup and Domain}

In this study air is the working fluid and obey's ideal gas law assumptions. The viscosity of the fluid is calculated using Sutherland's viscosity law. For better convergence and stability at the initial stage, the Courant number and relaxation factor were initialized with low values. Later, these values are increased gradually to a value of 10 and 0.8 respectively for smooth and fast solution convergence. The convergence level of residuals was brought down below $10^{-6}$ and simultaneously mass flow rate is also monitored. The inlet is specified as the pressure inlet and stagnation properties $\mathrm{P}_{0}$ and $\mathrm{T}_{0}$ are specified. The outlet boundary is considered as a non-reflecting pressure outlet boundary condition to specify static pressure or back pressure $(\mathrm{Pb})$. The Duct walls are designated as adiabatic and a no-slip boundary condition. The geometry along with the 
boundary condition is shown if Fig. 3(a) For the present numerical study, the inlet section height and width for a three-dimensional computational domain is $80 \times 80 \mathrm{~mm}$ and the four different half divergent angles $(\xi)$ are considered as $1^{\circ}, 2^{\circ}, 3^{\circ}$, and $4^{\circ}$. The domain divergence takes place only in the $y$ direction, and the width is constant throughout the duct. The length of the duct is $1500 \mathrm{~mm}$. Due to the symmetry of the problem to the channel centerline, the present study computes only 1 quarter of the flow field. The grid near the wall is clustered to resolve the boundary layer. The total number of grid points is around $2.5 \times 10^{6}$. The grid generated for a divergent duct is shown in Fig. 3(b). The accuracy of the solution mainly depends on grid cells and their distribution in the computational domain. The $\mathrm{y}+$ value for the present grid is maintained less than unity all over the domain.

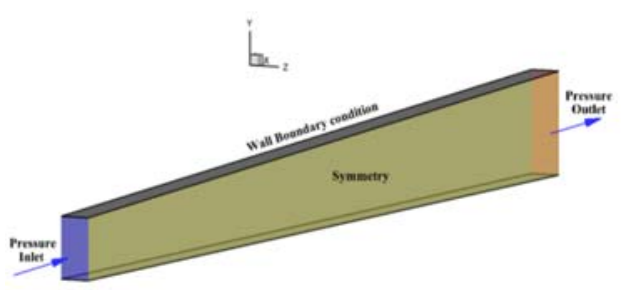

(a) Domain with boundary condition

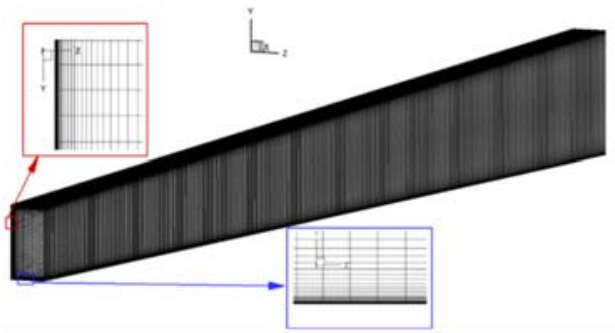

(b) Grid generated inside diverging duct

Fig. 3. Computation domain and Grid along symmetry plane $(\mathrm{Z} / \mathrm{W}=\mathbf{0})$.

\subsection{Grid Independence Test}

A grid independence study is conducted over a set of six grid resolutions for two dimensional flows. The number of cells in each grid is presented in Table 1. The solution should be independent of the grid resolution. A smaller number of cells will result in poor prediction of the shock boundary layer interaction. In the present study, the near wall mesh quality in all the grids is consistent so as to keep the wall $\mathrm{Y}^{+}$value lesser than 1 . The number of cells in the boundary layer region is 10 for grid 1 and 2, with cell numbers increasing to 15 and 20 for next set of 4 grids. The cell growth ratio from the wall is 1.35 to 1.1 for grids 1 to 6 , which was calculated after several iterative grid refinement processes along with turbulence model verification. The refinement of the grid results in a slight variation in the first shock location. The coarser grids display higher dissipation with a corresponding lower dissipation for the finer cell grids. A slight variation in the boundary layer parameter can cause a shock displacement. Figure 4 shows the Mach contour for three different grid refinements. The shock structure can be directly related to the grid density. The wall pressure and centerline pressure comparison for various grids is shown in Figs. 5(a) and 5(b) respectively. In the present analysis, the point at which the pressure steeply rise along the centerline is considered as the leading edge or starting point of the shock train. The shock location is observed for various grid resolutions and is plotted in Fig. 6. The variation in location is less than $1 \%$ for grid 4 to grid 6. Consequently, grid 4 is the minimum required grid resolution to capture macro-properties of pseudoshock system in a channel flow. Thus the grid 4 resolution criteria is maintained for all the cases.
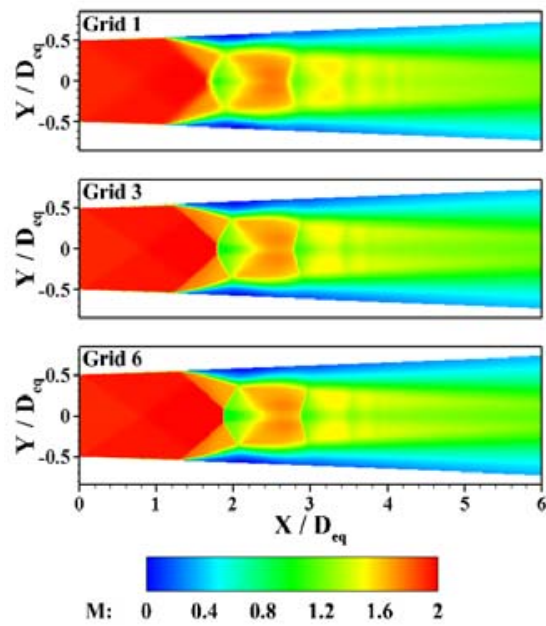

Fig. 4. Mach contour for various grid resolution.

Table 1 Number of grid points in each direction

\begin{tabular}{|c|c|c|c|c|c|c|}
\hline Grid & 1 & 2 & 3 & 4 & 5 & 6 \\
\hline$X_{\text {nodes }}$ & 350 & 800 & 1200 & 2000 & 2800 & 3500 \\
\hline$Y_{\text {nodes }}$ & 25 & 60 & 100 & 150 & 200 & 250 \\
\hline
\end{tabular}

\subsection{Verification of the Turbulence Model}

Modeling of turbulence in fluid flows is essential to evaluate a correct wall bounded turbulent flow with separation or an adverse pressure gradient. Most of the CFD codes utilize the RANS equation along with various turbulence closure model equations. Most of the closure models based on the Boussineq hypothesis consider the turbulent viscosity as an isotopic scalar quantity. With low computational cost it can perform well for predicting the mean values of a flow field. If the flow flow strongly depends on a turbulence parameter, models such as the LES (Large Eddy Simulation) or DNS (Direct Numerical Simulation) provide a solution. In the present work only macro properties which depend on geo-metrical and flow parameters are analyzed. To predict the generalized flow field structure in divergent channels two equation turbulence models, the $k-\varepsilon$ model, RNG $k-\varepsilon$ model, standard $k-\omega$ model 


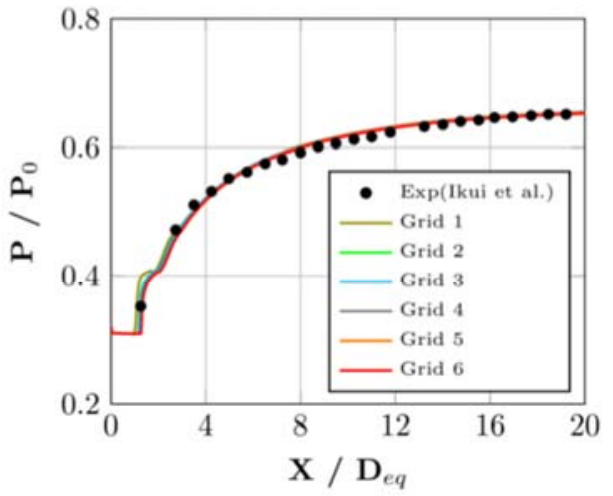

(a)

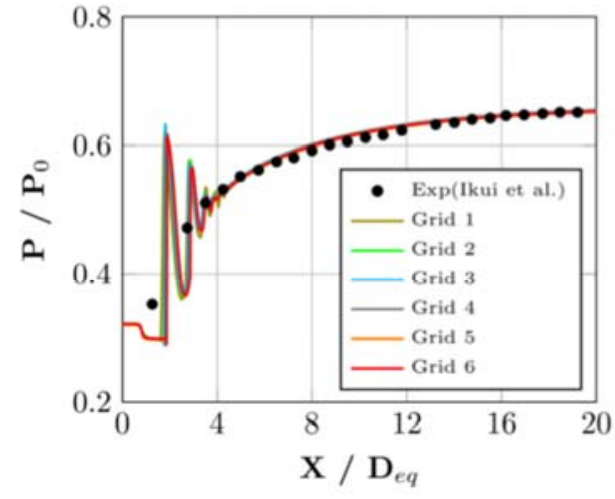

(b)

Fig. 5. Comparison of present numerical wall and centerline pressure data with experimental wall pressure data (Ikui et al. 1980).

and the SST model are used. Each turbulence model has its own requirement of mesh limits. Grid 3 is paired with the k- $\varepsilon$ model, RNG k- $\varepsilon$ model with enhanced wall treatment and grid 5 is used with the $k-\omega$ standard and SST model. The pseudo shock system captured in the divergent channel for various turbulence models is shown in Fig.7. The Standard $k-\varepsilon$ and RNG $k-\varepsilon$ model shows a larger core Mach stem in comparison with $k-\omega$ models. As discussed earlier, the $k-\varepsilon$ model is less sensitive for ad-verse pressure and separated flows. The SST $k$ - $\omega$ model shows clearer slip line behind the first shock wave. Figure 8 shows the pressure distribution for various turbulence models along the wall and the centerline. In comparison the SST $k-\omega$ model predictions are closer to the experimental value (Ikui et al. 1980) in terms of wall pressure distribution. Consequently all the present work simulations are carried out using the SST $k-\omega$ model.

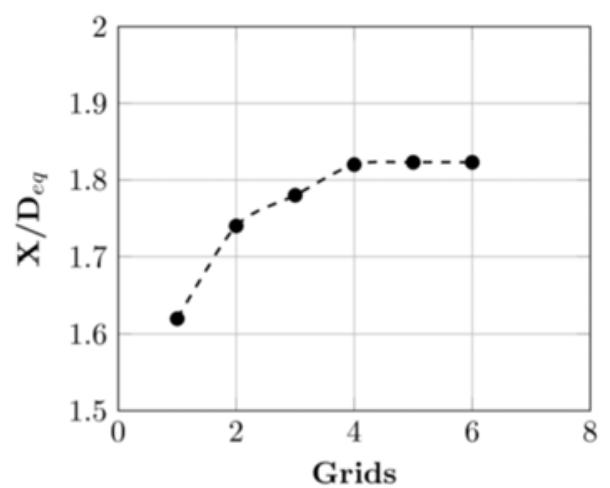

Fig. 6. Comparison of shock train leading edge location for various grids.

\section{RESULTS AND DISCUSSION}

The flow inside the isolator region of supersonic intake will help decelerate the flow from supersonic to subsonic by a series of compression waves. Usually the flow in a constant area duct will have a higher confinement ratio than a diverging duct. This is due to thickening of the boundary layer. The characteristics of pseudo shock wave differs from the constant area duct. The distance to observe a static pressure rise and the total pressure loss are significant to gain insight on designing the isolator. In the present work, an attempt has been made to understand the effect of back pressure, inflow Mach number and divergent angle on the characteristics of the shock train. Though the flow is three dimensional in nature, the center of the duct is assumed to be two-dimensional where the side wall does not influence the main flow field across the shock train (Gnani et al. 2018b). In a separate investigation, Section 3.4 shows the effect of side wall on the shock train. Table. 2 shows the case details in this research study.
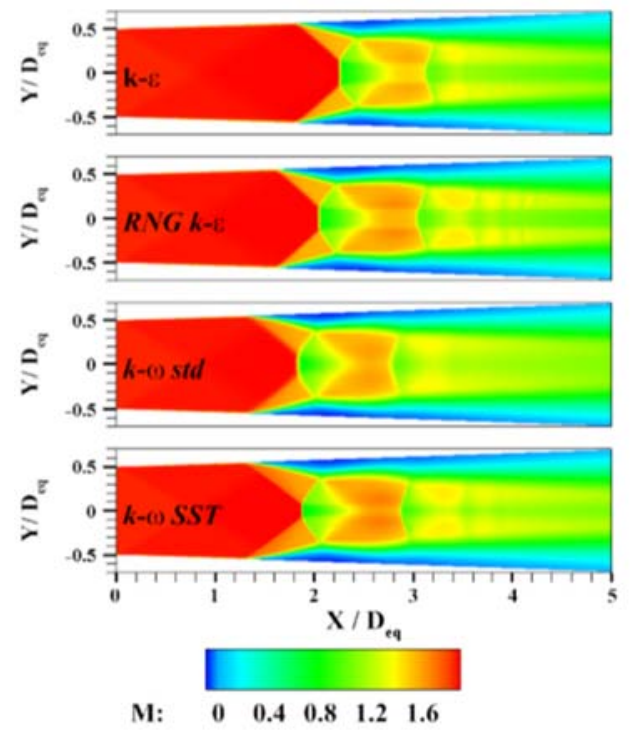

Fig. 7. Mach contour for various turbulence model effects. 


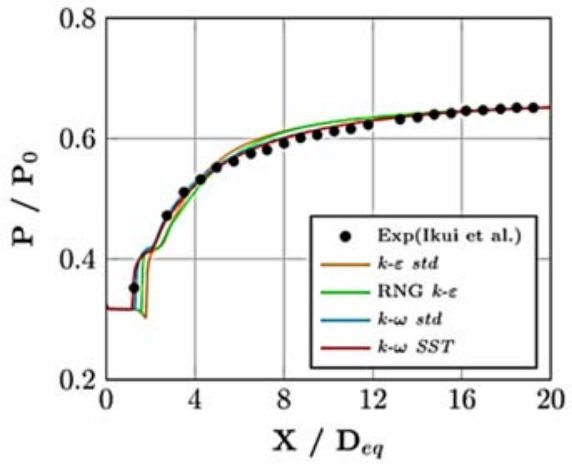

(a)

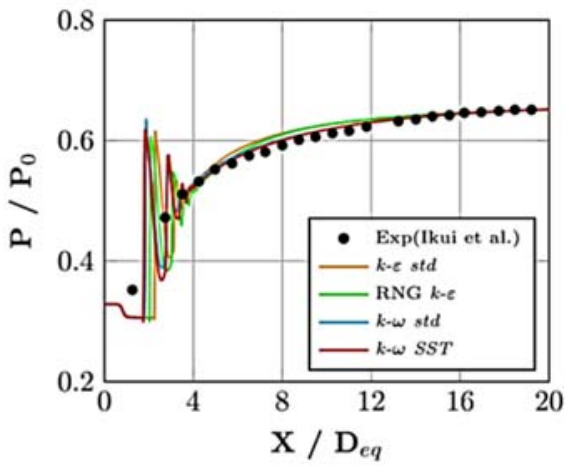

(b)

Fig. 8. Comparison of different turbulence model wall and centerline pressure data with experimental wall pressure data (Ikui et al. 1980).

Table 2 Input parameters for present cases

\begin{tabular}{|c|c|c|c|}
\hline Cases & $p_{b} / p_{0}$ & $\mathrm{M}$ & $\xi$ \\
\hline 1 & 0.65 & 1.8 & $2^{\circ}$ \\
\hline 2 & 0.59 & 1.8 & $2^{\circ}$ \\
\hline 3 & 0.52 & 1.8 & $2^{\circ}$ \\
\hline 4 & 0.50 & 1.6 & $2^{\circ}$ \\
\hline 5 & 0.50 & 1.8 & $2^{\circ}$ \\
\hline 6 & 0.50 & 2.0 & $2^{\circ}$ \\
\hline 7 & 0.46 & 2.0 & $1^{\circ}$ \\
\hline 8 & 0.46 & 2.0 & $2^{\circ}$ \\
\hline 9 & 0.46 & 2.0 & $3^{\circ}$ \\
\hline 10 & 0.46 & 2.0 & $4^{\circ}$ \\
\hline
\end{tabular}

\subsection{Effect of Back Pressure}

The effect of back pressure plays a major role in supersonic intake systems. For a stable combustion process, the isolator can provide a stable flow through a series of shock waves. At the same time the combustion process can elevate the back pressure which is transmitted upstream towards the inlet region. This can severely affect the intake performance by start/unstart condition. The major consideration in designing the supersonic intake is to have a minimum isolator length for a wide range of flow conditions. In this section, the effect of back pressure on shock train location and its characteristics are analyzed in a 2 deg half divergent channel with different back pressure. The details of flow conditions are presented in Table.2. Figure 9 shows Mach contour for three different back pressure levels $(100,90$ and $80 \mathrm{kPa})$. For case 1, the first shock appears to be a normal in the core region and in the other cases, due to flow expansion, the leading shock structure becomes oblique. The static pressure increase due to a single normal shock can be higher than the pressure increase due to a series of shock waves (Matsuo and Kim 1999a). Figure 10 shows the pressure distribution along the centerline and wall. The static pressure rise by a single normal shock $\left(\mathrm{P}_{\mathrm{s}}\right)_{\mathrm{n}}$ is shown by a dashed line. The pressure continues to rise in the area beyond the shock train due to channel divergence. Whereas for a constant area duct or channel, the pressure rise downstream of the shock train can be seen until the mixing region. Due to frictional losses the pressure drops slightly after the mixing region (Matsuo and Kim 1999a, Ikui et al. 1980). Thus the length of the pseudo-shock wave is the location with the maximum rise in pressure in case of constant area channel. For divergent channels it is obtained through the empirical correlation Eq. 1. For a constant upstream Mach number and Reynold's number the shock train length increases with a decrease in the back pressure and can be seen qualitatively from the x-density gradient contour shown in Fig.11. The shock structure and the distance between the first and second shock also varies with pressure downstream. It is also observed that the shock structure phenomenon changes from Mach reflection to regular reflection.

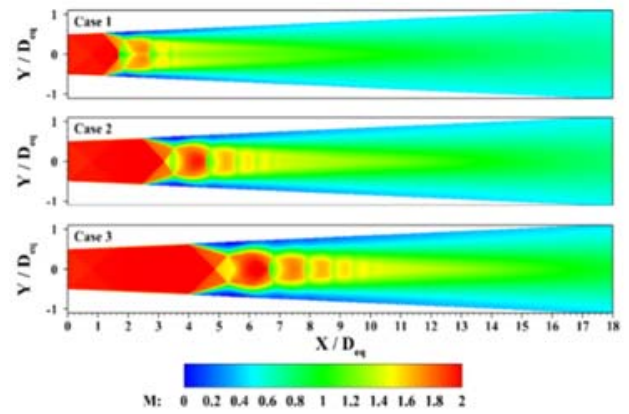

Fig. 9. Mach contour for different back pressure.

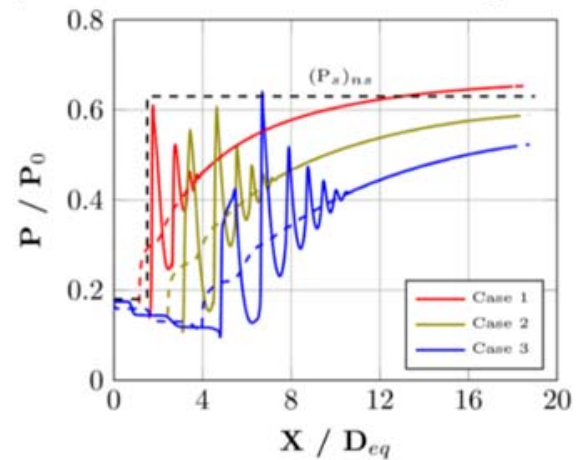

Fig. 10. Effect of varying a) back pressure and $b$ ) Mach number on pseudo shock wave in a $2 \mathrm{deg}$ divergent channel (solid lines - center line pressure, dashed lines - wall pressure). 
P. S. Vignesh Ram et al. / JAFM, Vol. 13, No. 4, pp. 1081-1092, 2020.
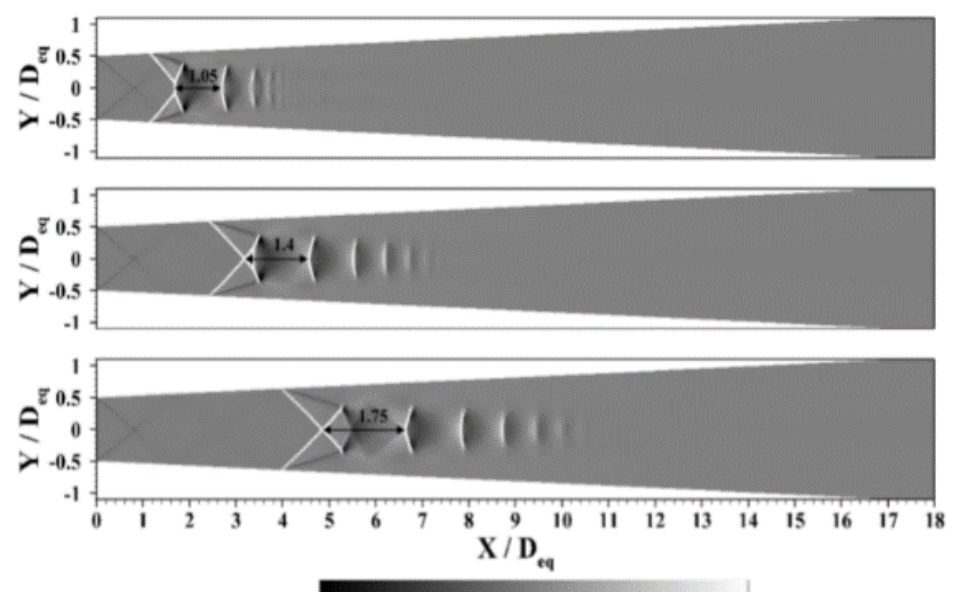

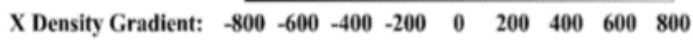

Fig. 11. $\mathrm{x}$ - density gradient contour for different back pressure.
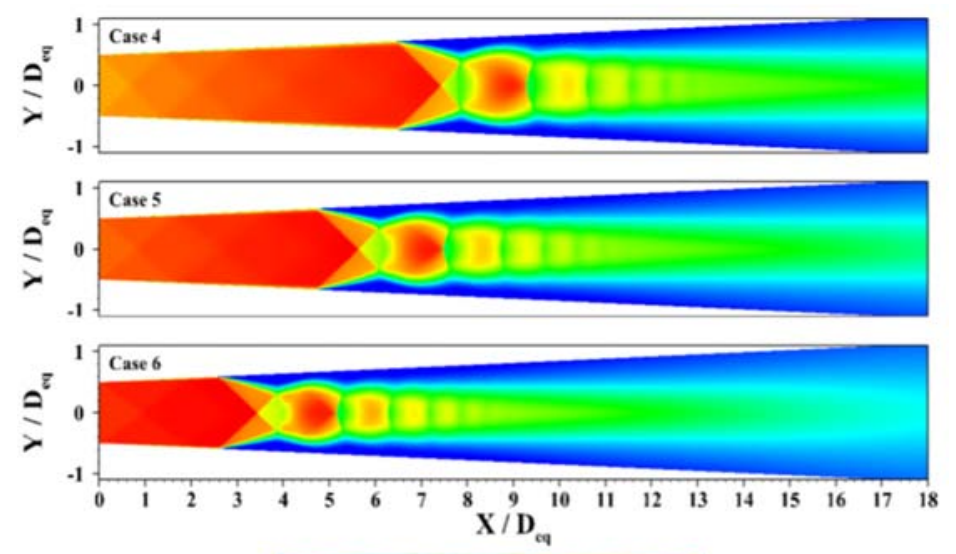

M: $\begin{array}{lllllllllll}0 & 0.2 & 0.4 & 0.6 & 0.8 & 1 & 1.2 & 1.4 & 1.6 & 1.8 & 2\end{array}$

Fig. 12. Effect of inlet Mach number variation.

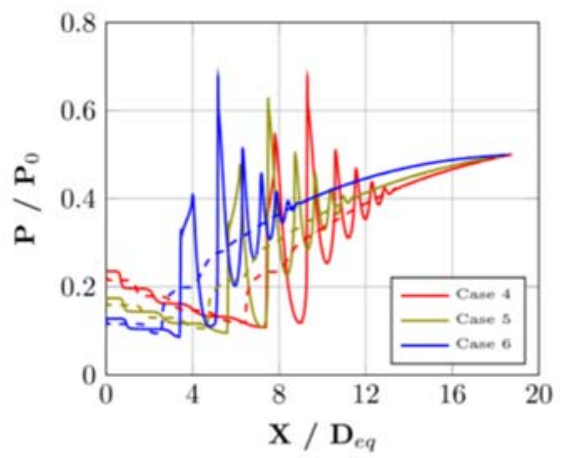

(a)

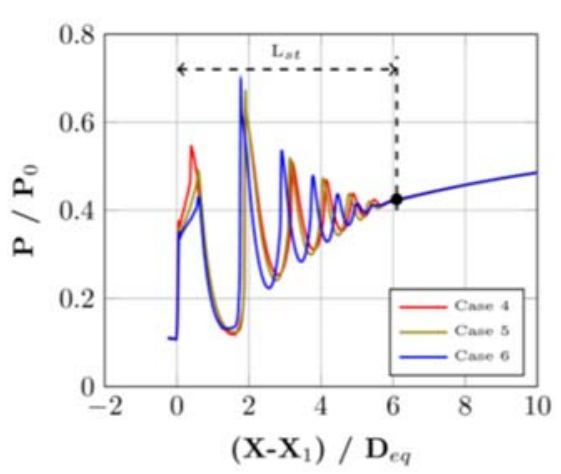

(b)

Fig. 13. Effect of varying Mach number on pseudo shock wave in a 2 deg divergent channel (a) centerline pressure (solid lines) and wall pressure (dashed lines) (b) centerline pressure with shifted $x$ location.

\subsection{Effect of Flow Mach Number}

In this section the influence of the free stream Mach number on the shock train location and its length are studied. The cases were simulated at stagnation pressure and stagnation temperature of $196 \mathrm{kPa}$ and $300 \mathrm{~K}$. The Reynold's number of the flow is $3 \times 10^{6}$. 


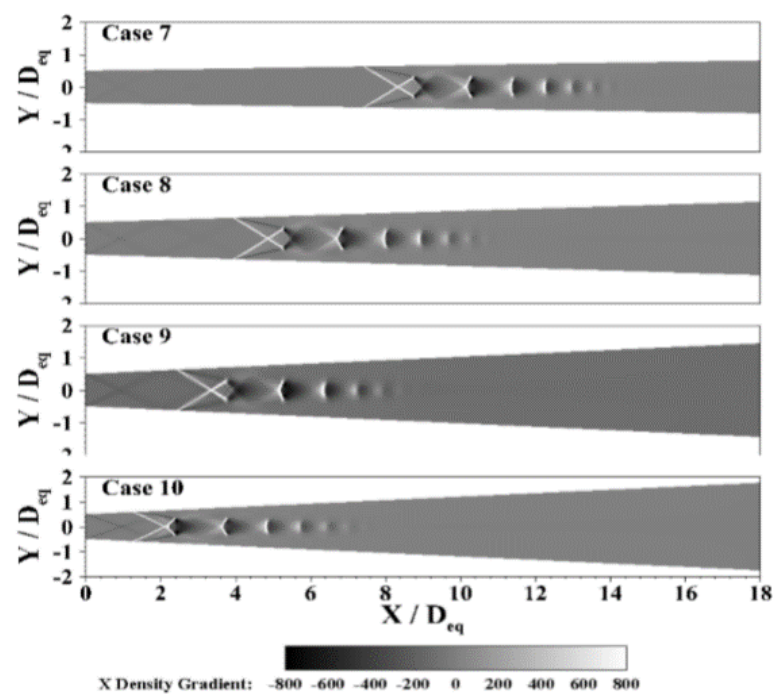

Fig. 14. $x$-Density gradient contour for different divergent angle.

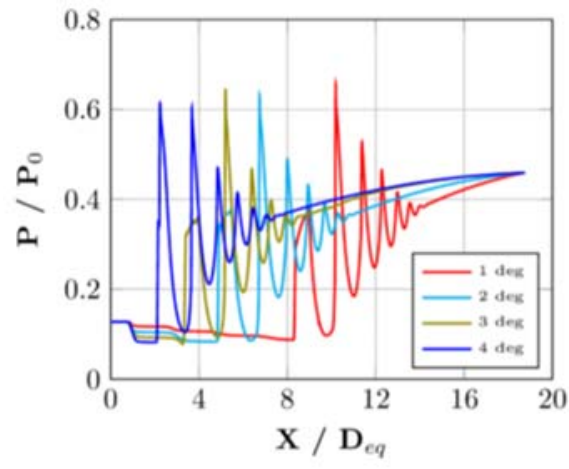

(a)

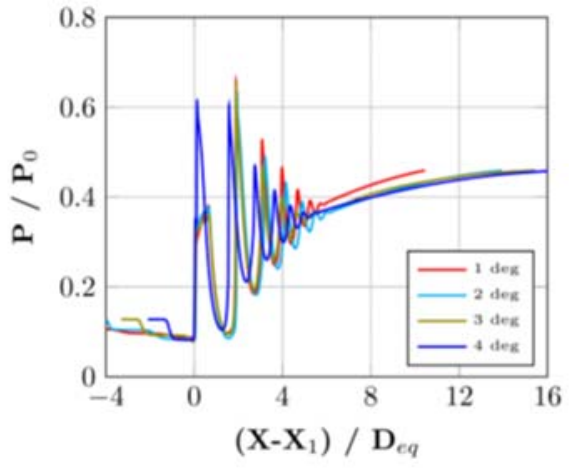

(b)

Fig. 15. Comparison of pressure ratio for different divergent angle a) along the centerline b) with shifted shock location.

By keeping the back pressure constant for all the three cases, we can observe from Fig.12 the shock train position moves upstream when increasing the freestream Mach number from 1.6 to 1.8. Weiss et al. (2010) has shown an increase in the Mach number will allow the shock train to move to a downstream position which is mainly due to the change in second throat height which in turns changes the back pressure. Weiss et al. (2010) also reported an increase in shock train length with a corresponding increase in the freestream Mach number. Conversely in the present simulation the change in shock train length is not observed. The reason behind the increase in length of the shock train in the experiment is only due to the change in back pressure. As this study presented in section 3.1 the variation in back pressure leads to an increase in shock train length. Figure 13(a) shows the pressure distribution along the centerline and wall for different freestream Mach numbers. Further-more the length of the shock train occurs in all three cases by shifting the leading shock location. Figure 13(b) demonstrates this shift when the starting shock location is 0 .

\subsection{Effect of Divergent Angle}

The scramjet isolator is designed either with a constant or small divergent angle to stabilize the flow before entering the combustion chamber. The growth of constant area duct boundary layer decreases the effective flow area while the length of the pseudo shock will increase with the confinement ratio (Matsuo and Kim 1999a, Gnani et al. 2016, Carroll and Dutton 1990). This narrows the range of the working pressure ratio in the isolator. In this section, the effect of the divergent angle on the shock train flow structure for fixed upstream and downstream conditions are discussed. The inlet stagnation pressure and stagnation temperature are $196 \mathrm{kPa}$ and $300 \mathrm{~K}$ respectively and the back pressure is $90 \mathrm{kPa}$. The half divergent angles $(\xi)$ considered for the present study are $1^{\circ}, 2^{\circ}, 3^{\circ}$, and $4^{\circ}$. Figure 14 shows the $\mathrm{x}$-density gradient contour for different divergent angles. The increase in the divergent angle causes the upstream movement of the shock train. The 


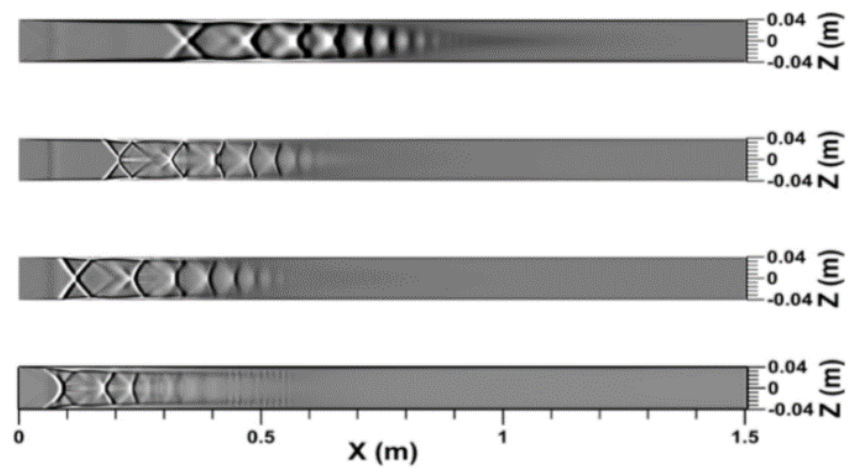

(a) Horizontal plane at $\mathrm{Y} / \mathrm{H}=0$ (from top $1^{\circ}, 2^{\circ}, 3^{\circ}$ and $4^{\circ}$ )

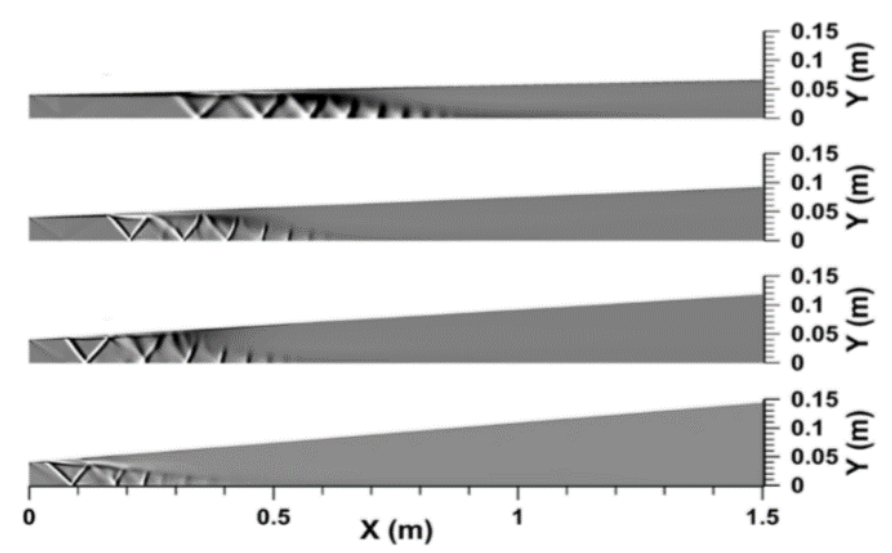

(b) Vertical plane at $\mathrm{Z} / \mathrm{W}=0$ (from top 1o, 2o, 3o and 4o)

Fig. 16. Numerical Schlieren for different divergent duct.

comparison of centerline pressure distribution for altered divergent channels are shown in Fig.15. It is observed that the $4^{\circ}$ channel has a small Mach stem near the center when all other angles produce an oblique shock pattern.

\subsection{Side Wall Effect on Shock Train}

The flow inside an isolator will be more complex in a three-dimensional flow field. In this section the effect of the side wall on the shock train is considered. The half divergent angles $(\xi)$ considered for the present study are $1^{\circ}, 2^{\circ}, 3^{\circ}$, and $4^{\circ}$ along with a constant width of $80 \mathrm{~mm}$. Simulated in a quarter domain. The inlet stagnation properties, $\mathrm{P} 0$ and $\mathrm{T} 0$ are $196 \mathrm{kPa}$ and $300 \mathrm{~K}$. The back pressure is specified as $90 \mathrm{kPa}$. Figures $16(\mathrm{a})$ and $16(\mathrm{~b})$ show the numerical Schlieren along $\mathrm{Y} / \mathrm{H}=0$ plane and $\mathrm{Z} / \mathrm{W}=$ 0 plane. The increase in divergent angle leads the pseudo shock wave to move upstream of the duct, but the presence of a side wall decreases the length of the pseudo-shock wave. This clearly shows that raising the level of confinement will lead to a larger pseudoshock length. Also in 2D cases it is noted that the flow separated after the first shock. By comparison, in 3D environments, the flow separates and reattaches downstream for divergent channels up to $3^{\circ}$ and a fully separated flow is only seen in the cases of $4^{\circ}$ divergent channels. Figure 17 shows the blockage ratio along the duct. Here in this paper the blockage ratio is given as,

$B_{1}=1-\left(1-2 \delta^{*} / D\right)^{2}$

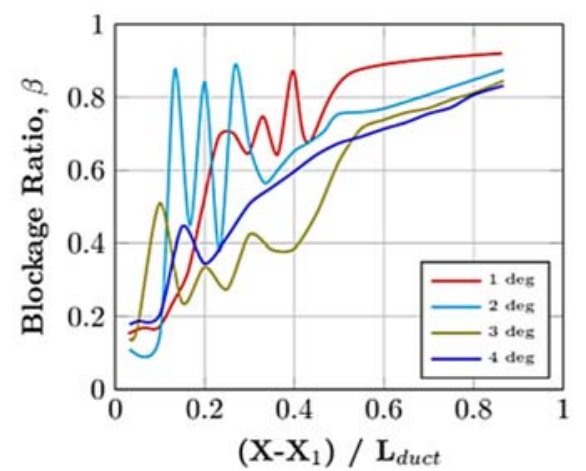

Fig. 17. Comparison of Blockage ratio along the duct.

As the divergent angle increases the flow separates producing a core flow representing the free jet flow showing an observed decrease in the blockage ratio. For $2^{\circ}$ duct the shock system is more attached to the top wall whereas, for the $8^{\circ}$ case it reaches near 


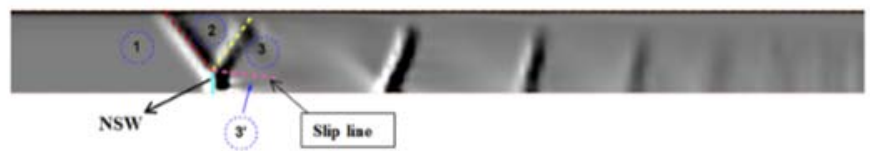

(a) $0 \mathrm{deg}$

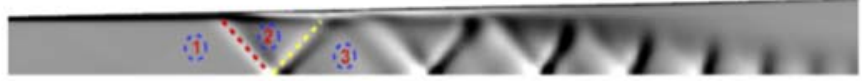

(b) 2 deg

Fig. 18. Shock structure comparison of constant area duct with 2 deg divergent duct.

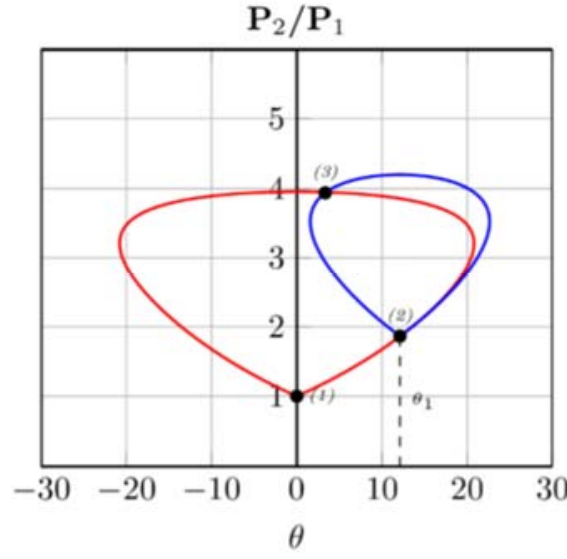

(a) 0 deg

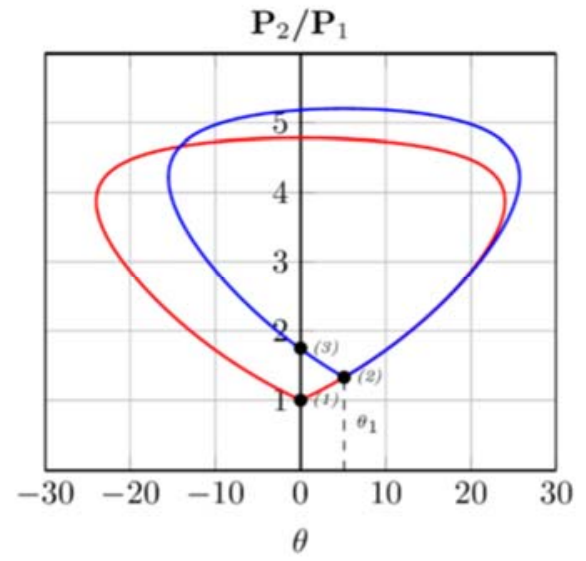

(b) 2 deg

Fig. 19. Comparison of pressure deflection diagram for con stant area and 20 divergent channel.

separation from the wall after the leading shock wave.

\subsection{Characteristic of Shock Train}

The characteristics of pseudo-shock waves can be better understood when comparing the structure obtained with the constant area duct (i.e. $\xi=0$ ). Figures 18(a) and 18(b) shows the x-density gradient contour of the shock structure inside a constant area duct and 2 deg diverging duct. A constant area duct with an upstream Mach number of less than 2.0 produces a normal shock train structure compared to Mach numbers beyond 2.0 producing an $\mathrm{x}$-shaped shock train (Matsuo and Kim 1999a; Carroll and Dutton 1990; Ikui et al. 1980). We can see that the presence of a normal shock wave (NSW) and oblique shock wave, though for Mach number lower than 2.2, an increase in the divergent angle motivates a transition from normal shock train to oblique shock train. To understand this variation for a constant Mach number $\mathrm{M}_{1}=2.0$, Figure 19 shows the deflection angle of an oblique shock as a function of the pressure ratio across the shock $\mathrm{p}_{2} / \mathrm{p}_{1}$. It is basically a derivation of the well-known pressuredeflection diagram. Matheis and Hickel (2015) explained the transition between regular and irregular shock patterns in the shock boundary layer interaction. The diagram consists of the $1^{\text {st }}$ deflection angle for the leading shock wave and depicts the changing flow direction downstream of the primary shock. When $\mathrm{p}_{2} / \mathrm{p}_{1}$ is smaller than 3.64 in the downstream flow, the oblique shock is supersonic when $\mathrm{M}_{2}>1$, yet when $\mathrm{p}_{2} / \mathrm{p}_{1}$ is higher, the shock strengthens and the flow downstream becomes subsonic when $\mathrm{M}_{2}<1$. The pre-shock Mach number in the constant area channel is 1.89 in the current observation. Here from Fig. 19(a) it is understood that a constant area channel at $\mathrm{M}=2.0$, the shock structure obtained is a Mach reflection phenomenon. In the case where two oblique shocks with the same shock Mach number interact symmetrically at the centerline, a regular reflection phenomenon occurs as shown in Fig. 19(b). For a divergent channel at M $=2.0$, the pre shock Mach number ahead of the Shock train is around 2.06. The pressure deflection diagram indicates that a regular reflection phenomenon occurs in a divergent duct. The change in the divergent angle also affects the characteristics of the shock structure phenomenon from a Mach Reflection to a Regular reflection. The total pressure loss due to a pseudo shock wave is shown in Fig. 20. It is higher for a steeper divergent angle. This loss is mainly due to separation which occurs downstream of the shock train region.

\section{CONCLUSION}

The numerical simulation is carried out to study the characteristics of the pseudo shock waves in a divergent channel at Mach 2.0. The effects of various 
flow parameters on the shock train are considered in a two-dimensional flow field. To check the accuracy of the present numerical simulation, a divergent duct of $2.2 \mathrm{deg}$ is validated against the experimental results shown in Ikui et al. (1980). The validation results are comparable with the experimental wall pressure data. Constant inlet stagnation properties and decreasing downstream pressure cause downstream movement of the shock train. Also the distance between the first shock and second shock increases. The downstream pressure disturbance changes the structure of the leading shock wave from a Mach reflection to a regular reflection phenomena. Changes to the inlet flow Mach number while maintaining constant stagnation properties, and with constant back pressure, do not produce an observable change in pseudo-shock length. The change in the inlet flow Mach number from 1.6 to 1.8 causes the upstream movement of the shock train. Likewise, the increase in angle causes up-stream movement of the shock train. The change in divergent angle in threedimensional flow with side walls causes the decrease in length of the shock train. In the case of twodimensional flow, the flow reattachment is not observed. Meanwhile, three-dimensional divergent channel flow shows that the flow separates and reattaches and then fully separates. Due to separated flow, the blockage ratio along the duct decreases with increases in the diver gent angle. From the pressure deflection diagram, the characteristics of pseudo shock wave are analyzed. In comparing the divergent duct with a constant area duct at $\mathrm{M}=2.0$, and stagnation pressure of $196 \mathrm{kPa}$ and Stagnation temperature of $300 \mathrm{~K}$, the results show a normal shock at the core flow and oblique shock in case of divergent duct. The pressure deflection diagram shows the transition of a Mach reflection to regular reflection when the duct divergent angle changes. The increase in divergent angle of the duct can also lead to increase in total pressure loss.

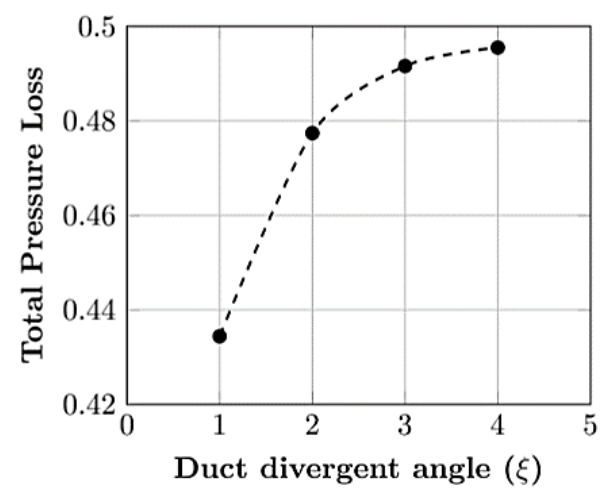

Fig. 20. Total pressure loss on duct divergent angle.

\section{ACKNOWLEDGMENTS}

This work was supported by the National Re-search Foundation of Korea (NRF) grant funded by the Korea government (MSIP) (No. NRF2016R1A2B3016436).

\section{REFERENCES}

Carroll, B. F. and J. C. Dutton (1990). Characteristics of multiple shock wave/turbulent boundarylayer interactions in rectangular ducts. Journal of Propulsion and Power 6(2), 186-193.

Chang, J., R. Zheng, L. Wang, W. Bao and D. Yu (2014). Backpressure unstart detection for a scramjet inlet based on information fusion. Acta Astronautica 95, 1-14.

Das, S. and J. K. Prasad (2010). Starting characteristics of a rectangular supersonic airintake with cowl deflection. The Aeronautical Journal (1968) 114(1153), 177-189.

Gnani, F., H. Zare-Behtash and K. Kontis (2016). Pseudo-shock waves and their inter-actions in high-speed intakes. Progress in Aerospace Sciences 82, $36-56$.

Gnani, F., H. Zare-Behtash, C. White and K. Kontis (2018a). Effect of back-pressure forcing on shock train structures in rectangular channels. Acta Astronautica 145, 471-481.

Gnani, F., H. Zare-Behtash, C. White and K. Kontis (2018b). Numerical investigation on threedimensional shock train structures in rectangular isolators. European Journal of Mechanics - B/Fluids 72, 586-593.

Gounko, Y. P. and I. I. Mazhul (2018, may). Flow turbulization in a pseudo-shock forming in an axisymmetric duct with a frontal inlet. Thermophysics and Aeromechanics 25(3), 347358.

Heiser W., D. Pratt, D. D. and U. Mehta (1994). Hypersonic Airbreathing Propulsion. AIAA Education Series, AIAA, Washington DC.

Huang, W., Z. guo Wang, M. Pourkashanian, L. Ma, D. B. Ingham, S. bin Luo, J. Lei and J. Liu (2011). Numerical investigation on the shock wave transition in a three-dimensional scramjet isolator. Acta Astronautica 68(11), 1669 1675.

Hutchins, K. E., M. R. Akella, N. T. Clemens, J. M. Donbar and S. Gogineni (2014). Experimental Identification of Transient Dynamics for Supersonic Inlet Unstart. Journal of Propulsion and Power 30(6), 1605-1612.

Ikui, T., K. Matsuo and K. Sasaguchi (1981, November). Modified diffusion model of pseudo-shock waves considering upstream boundary layers. Bulletin JSME 24(197), 19201927.

Ikui, T., K. Matsuo and M. Nagai (1974, June). The Mechanism of Pseudo-Shock Waves. Bulletin JSME 17(108), 731-739.

Ikui, T., K. Matsuo, H. Mochizuki and K. Somekawa (1980, January). Pseudo-Shock Waves in a Divergent Channel. Bulletin JSME 23(175), 20 25.

Kim, D. W., T. H. Kim and H. D. Kim (2017). A 
P. S. Vignesh Ram et al. / JAFM, Vol. 13, No. 4, pp. 1081-1092, 2020.

study on characteristics of shock train inside a shock tube. Theoretical and Applied Mechanics Letters 7(6), 366 - 371.

Matheis, J. and S. Hickel (2015). On the transition between regular and irregular shock patterns of shock-wave/boundary-layer interactions. Journal of Fluid Mechanics 776, 200-234.

Matsuo, K. Y. Miyazato and H. D. Kim (1999a, January). Shock train and pseudo-shock phenomena in internal gas Flows. Progress in Aerospace Sciences 35(1), 33-100.

Matsuo, K., Y. Miyazato and H. D. Kim (1999b) Mass-averaging pseudo-shock model in a straight flow passage. Proceedings of the Institution of Mechanical Engineers, Part G: Journal of Aerospace Engineering 213(6), 365375.

Menter, F. R. (1994). Two-equation eddy-viscosity turbulence models for engineering applications. AIAA Journal 32(8), 1598-1605.

Om, D. and M. E. Childs (1985). Multiple transonic shock-wave/turbulent boundary-layer interaction in a circular duct. AIAA Journal 23(10), 1506-1511.

Om, D., J. R. Viegas and M. E. Childs (1985) Transonic shock-wave/turbulent boundarylayer interactions in a circular duct. AIAA Journal 23(5), 707-714.

Quaatz, J., M. Giglmaier, S. Hickel and N. Adams (2014). Large-eddy simulation of a pseudoshock system in a Laval nozzle. International
Journal of Heat and Fluid Flow 49, 108 - 115. 8th Symposium on Turbulence \& Shear Flow Phenomena (TSFP8).

Sun, L. Q., H. Sugiyama, K. Mizobata and K. Fukuda (2003, May). Numerical and Experimental Investigations on the Mach 2 Pseudo-Shock Wave in a Square Duct. Journal of Visualization 6(4), 363-370.

Tamaki, T., Y. Tomita and R. Yamane (1970). A Study of Pseudo-Shock: 1st Report, $\lambda$-Type Pseudo-Shock. Bulletin of JSME 13(55), 51-58.

Tamaki, T., Y. Tomita and R. Yamane (1971). A Study of Pseudo-Shock: 2nd Re-port, X-Type Pseudo-Shock. Bulletin of JSME 14(74), 807817.

Wagner, J. L., K. B. Yuceil, A. Valdivia, N. T. Clemens and D. S. Dolling (2009). Experimental Investigation of Unstart in an Inlet/Isolator Model in Mach 5 Flow. AIAA Journal 47(6), 1528-1542.

Waltrup, P. and F. Billig (1973). Structure of Shock Waves in Cylindrical Ducts. AIAA Journal 11(10), 1404-1408.

Weiss, A., A. Grzona and H. Olivier (2010, January). Behavior of shock trains in a diverging duct. Experiments in Fluids 49(10), 355-365.

Zhang, Q. F., H. J. Tan, S. Sun, H. X. Bu and C. Y. Rao (2016). Unstart of a Hypersonic Inlet with Side Compression Caused by Downstream Choking. AIAA Journal 54(1), 28-38. 
